\title{
COMPORTAMENTO DE FASES DO SISTEMA BINARIO DIÓXIDO DE CARBONO + CUMARINA A ALTAS PRESSÕES
}

\author{
J. C. LIMA ${ }^{1}$, L. F. PINTO ${ }^{1}$, W. M. GIUFRIDA ${ }^{1}$, A. F. ZANETTE ${ }^{1}$, L. CARDOZO-FILHO ${ }^{1}$.
}

${ }^{1}$ Universidade Estadual de Maringá - Departamento de Engenharia Química

E-mail para contato: je.clima@hotmail.com

\begin{abstract}
RESUMO - Este trabalho tem por objetivo a investigação do comportamento de fases da mistura binária $\left\{\mathrm{CO}_{2}(1)+\right.$ Cumarina(2) $\}$ empregando o método estático sintético a partir uma célula de volume variável com visualização das transições de fases. Os experimentos foram conduzidos nas faixas de temperatura e fração molar de $298-338 \mathrm{~K}$ e $2 \times 10^{-3}-6 \times 10^{-3}$, respectivamente. As transições de fases ocorreram em pressões de até $20 \mathrm{MPa}$, sendo observados equilíbrios sólido-fluido (ESF), sólido-líquido-vapor (ESLV), líquido-vapor (ELV) e fluido-fluido (EFF).
\end{abstract}

\section{INTRODUÇÃO}

A cumarina (1,2-benzopiranona), formada por um anel aromático fundido em um anel lactona, possui grande potencial para utilização como herbicida (Oliveros-Bastidas et al., 2013), aromatizante (Oliveira et al., 2013), em perfumes, em cosméticos, anti-inflamatório (Leal et al., 2000), além de fins medicinais, como na prevenção e tratamento de edemas e câncer (Grötz et al., 2001; Mohler et al., 1994; Thornes et al., 1994).

A cumarina é encontrada em diversas plantas, como emburana, guaco, agrião, cominho e canela, e em frutas como morango, cereja, damasco e cumaru. Reporta-se na literatura que sua extração ocorre, normalmente, a partir do uso de solventes orgânicos, como etanol e metanol (Oliveros-bastidas et al., 2013; Thada et al., 2013). No entanto, a utilização de solventes orgânicos geram resíduos químicos e podem, inclusive, alterar as características do produto final devido às altas temperaturas empregadas durante os processos de extração e purificação. Contudo, conforme trabalhos anteriores, a extração da cumarina pode ser realizada por meio da utilização de gases pressurizados / supercríticos (Oliveira et al., 2013; Rodrigues et al., 2008). Essa tecnologia proporciona um maior rendimento na extração de princípios ativos e óleos por conta da característica de transporte e difusão desses gases quando estão em regiões supercríticas ou próximas a ela (Pinto et al., 2012, 2011). Conforme literatura, a manipulação da temperatura e da pressão pode mudar a seletividade da extração de determinados princípios ativos. Além dos significativos rendimentos observados neste tipo de extração (Garcia et al., 2012; Gonçalves et al., 2013; Lemos et al., 2012), uma grande vantagem dessa tecnologia é a produção de um princípio ativo livre de solventes residuais. $\mathrm{Na}$ literatura especializada são vários os trabalhos que reportam a utilização de gases $\left(\mathrm{CO}_{2}\right.$, propano e etileno) a altas pressões na extração de óleos e princípios ativos de oleaginosas (Carrara et al., 2011; Gonçalves et al., 2013; Jesus et al., 2013). 
Diante da possibilidade de utilização de fluidos supercríticos na extração da cumarina de diversas fontes vegetais, torna-se de extrema importância o estudo da solubilidade deste princípio ativo em fluidos supercríticos, visando assim, a determinação das condições ótimas de extração da cumarina.

(Hae Choi et al., 1998; Rodrigues et al., 2008) reportam em seus trabalhos o estudo da solubilidade da cumarina em dióxido de carbono supercrítico através da técnica dinâmica contínuo/extrativa. Contudo, uma das maiores dificuldades deste método é garantir que a fase rica no solvente supercrítico fique completamente saturada com o soluto. Além disso, esta técnica não permite o conhecimento das transições de fases. No entanto, a partir da obtenção do comportamento de transição de fases será possível aperfeiçoar processos de extração, reação e encapsulamento da cumarina em $\mathrm{CO}_{2}$ supercrítico.

Desta maneira, este trabalho tem como objetivo investigar o comportamento de fases do sistema binário $\left\{\mathrm{CO}_{2}(1)+\mathrm{Cumarina}(2)\right\}$ através do método estático sintético. Pois, através deste método é possível conhecer o comportamento das transições de fases do sistema.

\section{PROCEDIMENTO EXPERIMENTAL}

\subsection{Materiais.}

A cumarina $\left(\mathrm{C}_{9} \mathrm{H}_{6} \mathrm{O}_{2}\right)$ com pureza padrão $90 \%$ foi obtida pela Synth (Brasil). O dióxido de carbono (99.9 wt\% em fase líquida) foi obtido pela White Martins S.A. (Osasco, SP, Brasil).

\subsection{Aparato e procedimento experimental.}

O aparato e o procedimento experimental utilizado neste trabalho (Figura 1) foram descritos detalhadamente em trabalhos anteriores publicados por nosso grupo de trabalho (Rogério Favareto et al., 2008; Giufrida et al., 2011; Mazzer et al., 2012; Pinto et al., 2013; Prado et al., 2011; Thermodynamics et al., 2012). As medidas de transição de fases foram estudadas a partir do método estático sintético utilizando-se uma célula de volume varável provida de duas janelas de safira.

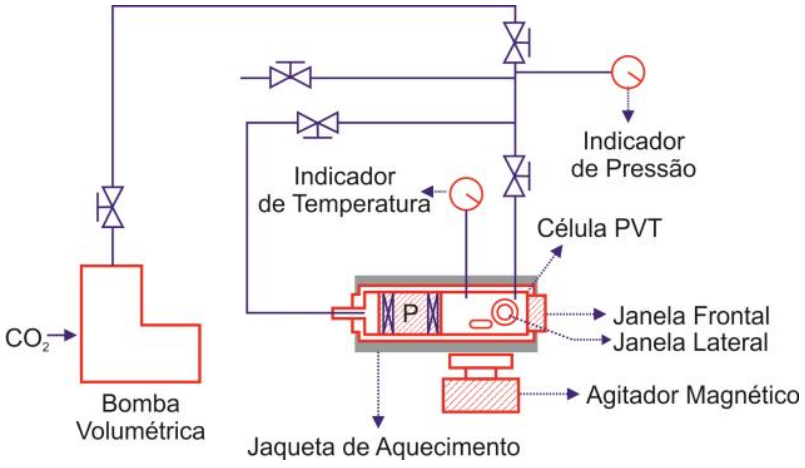

Figura 1. Aparato Experimental

O aparato (Figura 1) consiste em uma bomba do tipo seringa (ISCO, Model 260D), um banho termostatizado e uma célula de equilíbrio de volume variável (ajustado por meio da movimentação de um pistão) contendo duas janelas de safira (frontal e lateral) para 
observação do comportamento do sistema. Possui ainda, um transdutor de pressão (Smar, modelo LD 301), com incerteza de $\pm 0.01 \mathrm{MPa}$, para a aquisição das pressões de transição.

A célula de equilíbrio é provida de um pistão móvel que permite o controle de pressão dentro da célula. A mesma é equipada com uma jaqueta de alumínio e um controlador PID (Novus-N1100) conectado por um termopar (tipo T). Um indicador de temperatura (NovusN1100) conectado a um termopar (PT-100, com incerteza de $0,5 \mathrm{~K}$ ) foi colocado em contato direto com a mistura dentro da célula de equilíbrio. A quantidade de cumarina foi pesada em uma balança de precisão (Marte, modelo AM 220, incerteza: $0.0001 \mathrm{~g}$ ). A inserção de uma quantidade conhecida de $\mathrm{CO}_{2}$ foi realizada com o auxílio da bomba seringa, apresentando incertezas globais associadas às concentrações das misturas não maiores a $0.2 \%$, conforme metodologia de cálculo de Rodrigues-Reartes (Rodriguez-Reartes et al., 2009). A mistura é agitada por meio de um agitador magnético através de uma barra magnética revestida comteflon. Em seguida, o sistema é aquecido até temperatura desejada e pressurizada até surgimento de um sistema monofásico e permanece em agitação continua por 30 minutos. Após, o sistema é despressurizado a uma taxa de 0,1-0,2 $\mathrm{MPa} \mathrm{min}^{-1}$ até surgimento de um sistema bifásico ou trifásico. Esse procedimento é realizados três vezes, obtendo-se desvios padrões das pressões de transição na faixa de 0.01-0.9 MPa.

\section{RESULTADOS}

O aparato experimental e metodologia utilizada neste trabalho foram testados em trabalhos anteriores correspondendo ao estudo de equilíbrio de fases sólido+fluido ((Favareto et al., 2010; R. Favareto et al., 2008; Giufrida et al., 2014, 2011; Pinto et al., 2013). Favareto et al. (2010) mostrou que o aparato e o procedimento experimental são confiáveis na reprodução dos dados experimentais de solubilidade dos sistemas binários $\left\{\mathrm{CO}_{2}(1)+\right.$ naftaleno(2)\} e $\left\{\mathrm{CO}_{2}(1)+\right.$ bifenil(2) $\}$ reportados na literatura. Estes dados são apresentados na Figura 2.

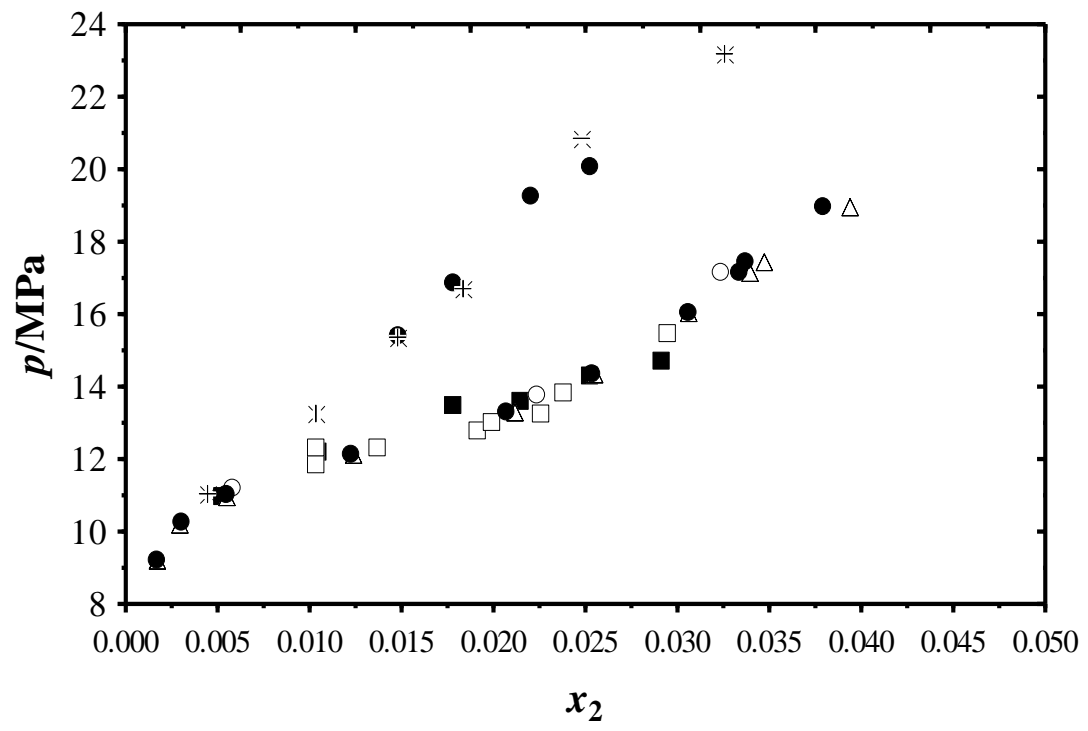

Figura 2. Diagrama pressão x composição para o sistema $\left\{\mathrm{CO}_{2}(1)+\right.$ naftaleno(2) $\}$ sob condições de equilíbrio sólido-vapor a $328 \mathrm{~K}$ ( Nosso grupo de pesquisa-Favareto et al. (2010)); $\Delta$ (McHugh and Paulaitis, 1980); $\bigcirc$ (Chang and Morrell, 1985); $\square$ (Zúñiga-Moreno et al., 2005) e sistema $\left\{\mathrm{CO}_{2}(1)+\right.$ bifenil(2) $\}$ sob condições de equilíbrio sólido - vapor a 328 K ( Nosso grupo de pesquisa - Favareto et al. (2010)); * (McHugh and Paulaitis, 1980). 
As isotermas de transição de fases obtidos para o sistema binário $\left\{\mathrm{CO}_{2}(1)+\right.$ Cumarina(2)\}, podem ser verificados na Figura 3. Conforme esta figura, função da fração molar do $\mathrm{CO} 2\left(x_{1}\right)$, é possível identificar os diversos tipos de comportamento de transição de fases obtidos em quatro isotermas $(298 \mathrm{~K}, 308 \mathrm{~K}, 318 \mathrm{~K}, 328 \mathrm{~K}$ e $338 \mathrm{~K})$. Contudo, observase que este sistema apresenta significativa imiscibilidade na isoterma de $298 \mathrm{~K}$, principalmente nas frações molares maiores de cumarina $(0,005$ e 0,006). Essa isoterma apresenta um comportamento do tipo crossover, visto que, seu comportamento cruza de maneira crescente todas as outras isotermas. Além disso, suas pressões de transição chegaram a valores próximos às maiores pressões de transição observadas para a isoterma de $338 \mathrm{~K}$. No entanto, este comportamento também é verificado na literatura (Hae Choi et al., 1998; Rodrigues et al., 2008). Nas isotermas de $298 \mathrm{~K}$ e $308 \mathrm{~K}$ foram observadas transições do tipo LV, SF, SFV e FF. Nas temperaturas mais elevadas, a partir de $318 \mathrm{~K}$, foi observado apenas transições do tipo fluido+fluido. Para este sistema também foram visualizados transições trifásicas SLV (sólido+líquido+vapor). Essas transições ocorreram em pressões próximas aos $6 \mathrm{MPa}$, mesmo em concentrações diferentes, tornando este tipo de transição independente da pressão.

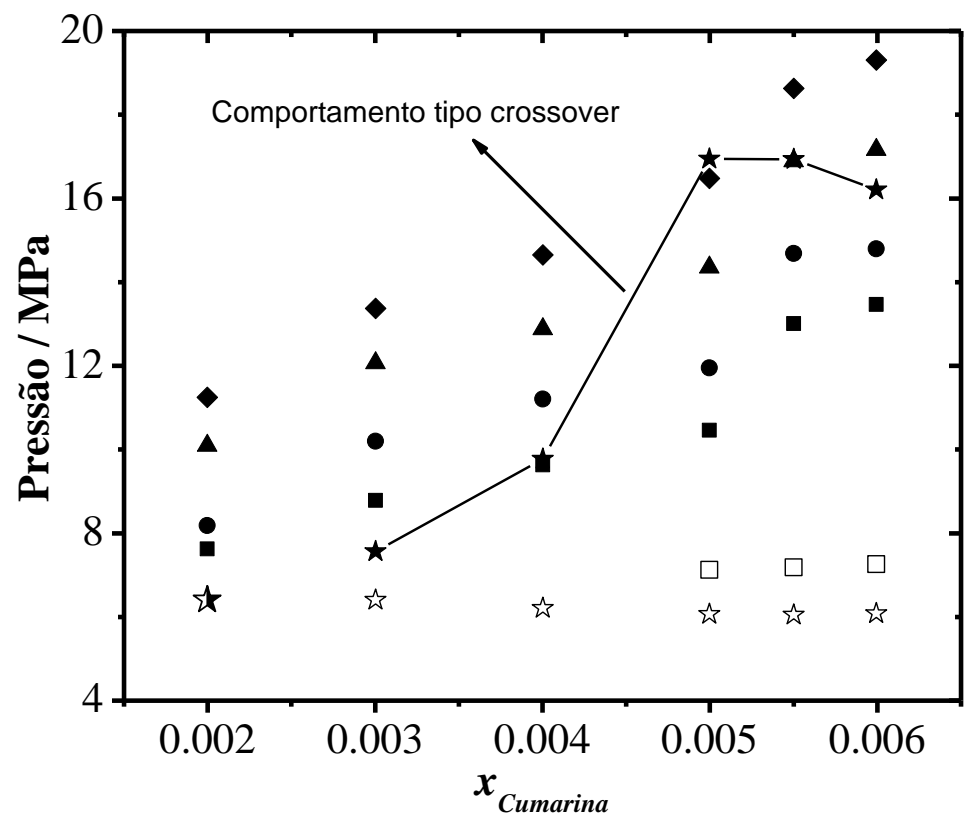

Figura 3 Diagrama de fases P-x do sistema $\left\{\mathrm{CO}_{2}(1)+\right.$ Cumarina (2) $\}$ em várias temperaturas (放: 298K - ESLV; $\star$ : 298 - ESF; ㅊ: 298K - ELV; $\square: 308 \mathrm{~K}-\mathrm{ESLV}$; $\mathbf{\square}: 308 \mathrm{~K}$

$-\mathrm{ESF} ; \bullet: 318 \mathrm{~K}-\mathrm{EFF} ; \boldsymbol{\Delta}: 328 \mathrm{~K}-\mathrm{EFF} ; \bullet: 338 \mathrm{~K}-\mathrm{EFF})$.

\section{CONCLUSÃO}

Este trabalho investigou o comportamento de fases do sistema binário $\left\{\mathrm{CO}_{2}(1)+\right.$ Cumarina(2) nas temperaturas de $298 \mathrm{~K}-338 \mathrm{~K}$ e pressões de até $20 \mathrm{MPa}$. As transições de fases observadas foram do tipo LV, FF, SF e SLV. Verificou-se na isoterma de $298 \mathrm{~K}$ um comportamento do tipo crossover. Ou seja, as pressões de transição nesta temperatura cruzam as pressões de transição das outras isotermas estudadas. Os dados experimentais aqui 
reportados são importantes informações na otimização de processos de extração, reação e encapsulamento da cumarina em $\mathrm{CO}_{2}$ supercrítico. 


\section{REFERENCIAS}

Carrara, V.D.S., Serra, L.Z., Cardozo-Filho, L., Cunha, E.F., Torres-Santos, E.C., Cortez, D.A.G. HPLC analysis of supercritical carbon dioxide and compressed propane extracts from Piper amalago L. with antileishmanial activity. Molecules 17, 15-33. doi:10.3390/molecules17010015, 2011.

Chang, H., Morrell, D.G. Solubilities of methoxy-1-tetralone and methyl nitrobenzoate isomers and their mixtures in supercritical carbon dioxide. J. Chem. Eng. Data 30, 74-78. doi:10.1021/je00039a025, 1985.

Favareto, R., Cabral, V.F., Corazza, M.L., Cardozo-Filho, L. Vapor-liquid and solid-fluid equilibrium for progesterone $+\mathrm{CO} 2$, progesterone+propane, and progesterone+n-butane systems at elevated pressures. J. Supercrit. Fluids 45, 161-170. doi:10.1016/j.supflu.2008.02.004, 2008.

Favareto, R., Fregadolli, P.H., Cabral, V.F., Antunes, O. a. C., Cardozo-Filho, L. Phase Equilibria of Acrylonitrile and p -Bromobenzaldehyde in Carbon Dioxide. J. Chem. Eng. Data 53, 1080-1084. doi:10.1021/je700448v, 2008.

Favareto, R., Pereira, J.R.D., Santana, C.C., Madureira, E.H., Cabral, V.F., Tavares, F.W., Cardozo-Filho, L. High-pressure phase diagram of the drug mitotane in compressed and/or supercritical CO2. J. Chem. Thermodyn. 42, 286-290. doi:10.1016/j.jct.2009.08.017, 2010.

Garcia, V.A.D.S., Cabral, V.F., Zanoelo, É.F., da Silva, C., Filho, L.C. Extraction of Mucuna seed oil using supercritical carbon dioxide to increase the concentration of 1-Dopa in the defatted meal. J. Supercrit. Fluids 69, 75-81. doi:10.1016/j.supflu.2012.05.007, 2012.

Giufrida, W.M., Pinto, L.F., Zanette, A.F., Voll, F.A.P., Kunita, M.H., Cabral, V.F., CardozoFilho, L. Liquid-vapor equilibrium data of $\mathrm{CO} 2+$ dichloromethane+medroxyprogesterone system. Fluid Phase Equilib. 362, 307-312. doi:10.1016/j.fluid.2013.10.037, 2014.

Giufrida, W.M., Rodriguez-Reartes, S.B., Alonso, C.G., Zabaloy, M.S., Cabral, V.F., Tavares, F.W., Cardozo-Filho, L. High-Pressure Experimental Data of CO 2 + Mitotane and CO 2 + Ethanol + Mitotane Mixtures. J. Chem. Eng. Data 56, 4333-4341. doi:10.1021/je101233k, 2011.

Gonçalves, R.M., Lemos, C.O.T., Leal, I.C.R., Nakamura, C.V., Cortez, D.A.G., da Silva, E.A., Cabral, V.F., Cardozo-Filho, L. Comparing conventional and supercritical extraction of (-)-mammea $\mathrm{A} / \mathrm{BB}$ and the antioxidant activity of Calophyllum brasiliense extracts.

Molecules 18, 6215-29. doi:10.3390/molecules18066215, 2013.

Grötz, K.A., Wüstenberg, P., Kohnen, R., Zepelin, H.H. Prophylaxis of radiogenic sialadenitis and mucositis by coumarin / troxerutine in patients with head and neck cancer - a prospective , randomized , placebo-controlled, double-blind study 34-39. doi:10.1054/bjom.2000.0459, 2001. 
Hae Choi, Y., Kim, J., Jeong Noh, M., Sun Choi, E., Yoo, K.-P. Effect of functional groups on the solubilities of coumarin derivatives in supercritical carbon dioxide. Chromatographia 47, 93-97. doi:10.1007/BF02466793, 1998.

Jesus, A.A., Almeida, L.C., Silva, E.A., Filho, L.C., Egues, S.M.S., Franceschi, E., Fortuny, M., Santos, A.F., Araujo, J., Sousa, E.M.B.D., Dariva, C. Extraction of palm oil using propane, ethanol and its mixtures as compressed solvent. J. Supercrit. Fluids 81, 245-253. doi:10.1016/j.supflu.2013.06.011, 2013.

Leal, L.K., Ferreira, a a, Bezerra, G. a, Matos, F.J., Viana, G.S. Antinociceptive, antiinflammatory and bronchodilator activities of Brazilian medicinal plants containing coumarin: a comparative study. J. Ethnopharmacol. 70, 151-9, 2000.

Lemos, C.O.T., Garcia, V.A.D.S., Gonçalves, R.M., Leal, I.C.R., Siqueira, V.L.D., Filho, L.C., Cabral, V.F. Supercritical extraction of neolignans from Piper regnelli var. pallescens. J. Supercrit. Fluids 71, 64-70. doi:10.1016/j.supflu.2012.07.003, 2012.

Mazzer, H.R., Santos, J.C.O., Cabral, V.F., Dariva, C., Kunita, M.H., Rubira, A.F., Aznar, M., Cardozo-Filho, L. Phase Behavior at High Pressure of the Ternary System: CO2, Ionic Liquid and Disperse Dye. J. Thermodyn. 2012, 1-6. doi:10.1155/2012/921693, 2012.

McHugh, M., Paulaitis, M.E. Solid solubilities of naphthalene and biphenyl in supercritical carbon dioxide. J. Chem. Eng. Data 25, 326-329. doi:10.1021/je60087a018, 1980.

Mohler, J.L., Williams, B.T., Thompson, I.M., Marshall, M.E. Coumarin (1,2-benzopyrone) for the treatment of prostatic carcinoma. J. Cancer Res. Clin. Oncol. 120 Suppl, S35-8, 1994.

Oliveira, A.L., Pozza, L.N.L., Santos, D.N., Kamimura, E.S., Vicente, E., Cabral, F.A. Supercritical extraction of coumarin from guaco (Mikania laevigata and Mikania glomerata) for pharmaceutical applications. J. Supercrit. Fluids 83, 65-71.

doi:10.1016/j.supflu.2013.07.019, 2013.

Oliveros-bastidas, A.D.J., Demuner, A.J., Barbosa, L.C. de A. Chemical Characterization by GC-MS and Phytotoxic Potential of non-polar and polar fractions of seeds of Dipteryx odorata (Aubl.) Wild. from Venezuelan regions 36, 502-506, 2013.

Pinto, L.F., da Silva, D.I.S., Rosa da Silva, F., Ramos, L.P., Ndiaye, P.M., Corazza, M.L. Phase equilibrium data and thermodynamic modeling of the system (CO2+biodiesel+methanol) at high pressures. J. Chem. Thermodyn. 44, 57-65. doi:10.1016/j.jct.2011.07.019, 2012.

Pinto, L.F., Ndiaye, P.M., Ramos, L.P., Corazza, M.L. The Journal of Supercritical Fluids Phase equilibrium data of the system CO $2+$ glycerol + methanol at high pressures. J. Supercrit. Fluids 59, 1-7. doi:10.1016/j.supflu.2011.08.002, 2011.

Pinto, L.F., Rodriguez-Reartes, S.B., Corazza, M.L., Cabral, V.F., de Araújo, P.H.H., Madureira, E.H., Zabaloy, M.S., Cardozo-Filho, L. Phase behavior of carbon 
dioxide+medroxyprogesterone acetate system at high pressures. Fluid Phase Equilib. 349, 111. doi:10.1016/j.fluid.2013.03.019, 2013.

Prado, I.M., Giufrida, W.M., Alvarez, V.H., Cabral, V.F., Quispe-Condori, S., Saldaña, M.D. a., Cardozo-Filho, L. Phase Equilibrium Measurements of Sacha Inchi Oil (Plukenetia volubilis) and CO2 at High Pressures. J. Am. Oil Chem. Soc. 88, 1263-1269. doi:10.1007/s11746-011-1786-z, 2011.

Rodrigues, R.F., Tashima, A.K., Pereira, R.M.S., Mohamed, R.S., Cabral, F. a. Coumarin solubility and extraction from emburana (Torresea cearensis) seeds with supercritical carbon dioxide. J. Supercrit. Fluids 43, 375-382. doi:10.1016/j.supflu.2007.07.014, 2008.

Rodriguez-Reartes, S.., Cismondi, M., Franceschi, E., Corazza, M.L., Oliveira, J.V., Zabaloy, M.S. The Journal of Supercritical Fluids High-pressure phase equilibria of systems carbon dioxide + n-eicosane and propane + n-eicosane 50, 193-202. doi:10.1016/j.supflu.2009.06.017, 2009.

Thada, R., Chockalingam, S., Dhandapani, R.K., Panchamoorthy, R. Extraction and quantitation of coumarin from cinnamon and its effect on enzymatic browning in fresh apple juice: a bioinformatics approach to illuminate its antibrowning activity. J. Agric. Food Chem. 61, 5385-90. doi:10.1021/jf4009789, 2013.

Thermodynamics, J.C., Santos, J.C., Mazzer, H.R., Machado, G.D., Andreaus, J., Cabral, V.F., Zabaloy, M.S., Cardozo-filho, L. High-pressure phase behaviour of the system ( CO $2+$ C . I . Disperse Orange 30 dye ). J. Chem. Thermodyn. 48, 284-290. doi:10.1016/j.jct.2011.12.040, 2012.

Thornes, R.D., Daly, L., Lynch, G., Breslin, B., Browne, H., Browne, H.Y., Corrigan, T., Daly, P., Edwards, G., Gaffney, E., Henley, J., Healy, T., Keane, F., Lennon, F., Mcmurray, N., Loughlin, S.O., Shine, M., Tanner, A. Treatment with coumarin to prevent or delay recurrence of malignant melanoma 120, 32-34, 1994.

Zúñiga-Moreno, A., Galicia-Luna, L.A., Camacho-Camacho, L.E. Measurements of solid solubilities and volumetric properties of naphthalene+carbon dioxide mixtures with a new assembly taking advantage of a vibrating tube densitometer. Fluid Phase Equilib. 234, 151163. doi:10.1016/j.fluid.2005.06.010, 2005. 